\title{
Differential Modulation of Long-Term Depression by Acute Stress in the Rat Dorsal and Ventral Hippocampus
}

\author{
Nicola Maggio and Menahem Segal \\ Department of Neurobiology, The Weizmann Institute, 76100 Rehovot, Israel
}

The ventral hippocampus $(\mathrm{VH})$ was recently shown to express lower-magnitude long-term potentiation (LTP) than the dorsal hippocampus (DH). An exposure to acute stress reversed this difference, and VH slices from stressed rats expressed larger LTP than controls, whereas LTP in the DH was suppressed by stress. We have now used long-term depression (LTD)-generating trains of stimulation to examine whether this differential LTP reflects a genuine difference in synaptic modifiability between the two sectors of the hippocampus. Surprisingly, slices of DH and VH express similar magnitudes of LTD. However, while prior stress enhanced LTD in the DH, it actually converted LTD to slow-onset, robust LTP in the VH. These two effects of stress on LTD were blocked by glucocorticosterone receptor (GR) and mineralocorticosterone receptor (MR) antagonists, respectively. Acute exposure of slices to a GR agonist dexamethasone facilitated LTD in slices of both DH and VH, while activation of MRs by aldosterone converted LTD to LTP in both regions. Thus, differential activation of the two species of corticosterone receptors determines the ability of the two sectors of the hippocampus to undergo plastic changes in response to LTD-inducing stimulation.

\section{Introduction}

Recent findings propose that a functional differentiation exists along the septotemporal axis of the hippocampus, such that cognitive functions, traditionally assigned to this structure, are, in fact, associated mainly with its dorsal sector (Colombo et al., 1998; Moser and Moser, 1998; Strange et al., 1999; Pothuizen et al., 2004). In contrast, the ventral hippocampus $(\mathrm{VH})$ has been associated with regulation of anxiety and is assumed to exert its effects mainly through connections with the amygdala and hypothalamus (Kjelstrup et al., 2002; Bannerman et al., 2003, 2004; Degroot and Treit, 2004; Trivedi and Coover, 2004). A major difference between the dorsal hippocampus (DH) and VH is in the ability to evoke long-term potentiation (LTP) of reactivity to afferent stimulation (Papatheodoropoulos and Kostopoulos, 2000; Maruki et al., 2001; Colgin et al., 2004; Maggio and Segal, 2007a). Specifically, LTP in VH is significantly lower than that elicited in the $\mathrm{DH}$.

We found that following exposure to acute swim stress, the activation of mineralocorticosterone receptors (MRs) produced an enhancement of LTP in VH, while in DH, LTP was impaired by activation of glucocorticosterone receptors (GRs) (Maggio and Segal, 2007b). These studies suggest that MRs and GRs assume opposite roles in regulation of synaptic plasticity following an acute exposure to stressors.

Using hippocampal slices, we explored whether the reduced LTP in the VH reflects a genuine reduction in overall ability to express plasticity, and thus will also be reflected in a reduced ability to express long-term depression (LTD), or reflects a dif-

Received April 22, 2009; revised May 21, 2009; accepted May 22, 2009.

This work was supported by a grant from the German Research Foundation (DIP, L-1922).

Correspondence should be addressed to Nicola Maggio at the above address. E-mail: nicola.maggio@weizmann.ac.il.

D0I:10.1523/JNEUROSCI.1901-09.2009

Copyright $\odot 2009$ Society for Neuroscience $\quad$ 0270-6474/09/298633-06\$15.00/0 ferent modification threshold, such that the VH will express a larger LTD for the same stimulation frequency (Bear et al., 1987). Subsequently, we examined whether stress has any effect on LTD generation in the two sectors of the hippocampus.

\section{Materials and Methods}

Animals. Experiments were conducted according to institutional rules and regulations. Young adult 10- to 12 -week-old male Wistar rats were used. The acute swim stress protocol consisted of exposing a naive rat to a forced swim for $15 \mathrm{~min}$ in a circular water tank (diameter, $0.5 \mathrm{~m}$; height, $0.5 \mathrm{~m}$ ). Water depth was $40 \mathrm{~cm}$, and temperature was maintained at $23 \pm$ $1^{\circ} \mathrm{C}$. Stress exposure was always commenced at 9:00 A.M., when the plasma corticosterone levels are low (Finn et al., 2003, Krugers et al., 2005).

Drugs. The MR antagonist spironolactone (Sigma) and the GR antagonist RU38486 (mifepristone) were dissolved in 1,2-propandiole (20 $\mathrm{mg} / 10 \mathrm{ml}$ ) and were injected subcutaneously $(20 \mathrm{mg} / \mathrm{kg}$ ) (Smriga et al., 1998) $1 \mathrm{~h}$ before the forced swim.

The behavior of a subset of animals ( $n=4$ in each group) in the water tank was measured to determine whether the drugs had any effect on immobility. Surprisingly, while the control, GR-blocked, and GR/MRblocked rats spent approximately the same time being immobile, the MR-blocked rats spent significantly more time being immobile in the water tank (supplemental Fig. 1, available at www.jneurosci.org as supplemental material). The relevance of this observation will be discussed below.

Slice procedures. Slice procedures were as detailed previously (Maggio and Segal, 2007b). Briefly, the rat was rapidly decapitated, the hippocampus was removed, and $350 \mu \mathrm{m}$ slices were prepared using a McIlwain tissue slicer. Slices were collected from the $2 \mathrm{~mm}$ dorsal and ventral poles of the hippocampus. Slices were perfused with artificial CSF (containing, in mM, $124 \mathrm{NaCl}, 2 \mathrm{KCl}, 26 \mathrm{NaHCO}_{3}, 1.24 \mathrm{KH}_{2} \mathrm{PO}_{4}, 2.5 \mathrm{CaCl}_{2}, 2 \mathrm{MgSO}_{4}$, and 10 glucose, $\mathrm{pH}$ 7.4) in a standard interface chamber. Recordings were made with a glass pipette containing $0.75 \mathrm{M} \mathrm{NaCl}(4 \mathrm{M})$ placed in the stratum radiatum CA1. Stimulation was delivered through two sets of bipolar nichrome electrodes placed on both sides of stratum radiatum, equidistant from the recording electrode. LTD was induced by applying 900 stimuli at $1 \mathrm{~Hz}$ [as in the study by Dudek and Bear (1992)] with 
baseline values recorded at a frequency of 0.016 $\mathrm{Hz}$ at a stimulation intensity producing $50 \%$ of maximal EPSPs. Values for each slice were averaged across a 5 min interval, and the results of at least seven slices of each treatment were averaged. EPSP slope changes following the LTD induction protocol were calculated with respect to baseline. There were no systematic differences in the magnitudes of the baseline responses in the different conditions.

\section{Results}

LTP is lower in CA1 of the VH than in $\mathrm{DH}$, but LTD is similar in both sectors

As shown previously (Maggio and Segal, 2007b), baseline input-output relations were the same in the $\mathrm{DH}$ and $\mathrm{VH}$, but slices from the $\mathrm{VH}$ were unable to produce as large LTP as its DH counterpart (Fig. 1A). At 40 min after a tetanic stimulation, the level of potentiation reached $174 \pm 4.7 \%$ of baseline level in $\mathrm{DH}$, whereas in slices of the $\mathrm{VH}$, this potentiation reached $129 \% \pm 5.9$ of baseline levels ( $n=7$ slices in each group, $p<0.01)$.

In a separate set of slices, a lowfrequency stimulation $[1 \mathrm{~Hz}, 900$ pulses, or two successive trains of 600 pulses at 1 $\mathrm{Hz}$ each (as in supplemental Fig. 2, available at www.jneurosci.org as supplemental material)] produced a similar LTD, in the two sectors of the hippocampus, amounting to $85 \pm 7 \%$ of baseline level in the $\mathrm{DH}$ slices, and $81 \pm 6.9 \%(p=0.89)$ of baseline level in VH slices. These results indicate that LTD is not a simple reflection of LTP with respect to the DH/VH disparity.

Acute swim stress alters LTP and LTD in both $\mathrm{DH}$ and $\mathrm{VH}$

Following an acute stress, the two sectors of the hippocampus reacted in an opposite manner: LTP in the DH was reduced to $147 \pm 5.7 \%$ of baseline, while VH LTP was now enhanced to $187 \pm 5.7 \%(p<0.001$ compared with control unstressed values) (Fig. $1 B$ ), as seen previously (Maggio and Segal, 2007b). Even more striking, LTD in $\mathrm{DH}$ was enhanced, amounting to $49.4 \pm$ $6.5 \%$ of baseline level $(p<0.01$ compared with control, unstressed condition) (Fig. $1 B)$, whereas LTD was reversed into a slowonset LTP in the VH, amounting to $125 \pm$ $7 \%$ above baseline at $40 \mathrm{~min}$ after the exposure to the LTD-generating protocol $(p<$ 0.001 compared with control, unstressed condition). The effect of the stress on LTD was even more striking when the LTD-generating protocol consisted of two consecutive trains of 600 pulses at $1 \mathrm{~Hz}$ (supplemental Fig. $2 \mathrm{~A}, \mathrm{~B}$, available at www.jneurosci.org as supplemental material).

Pharmacology of stress-induced LTD changes

We then examined whether this striking difference in behavior of $\mathrm{DH}$ and $\mathrm{VH}$ slices in response to stress is mediated by activation

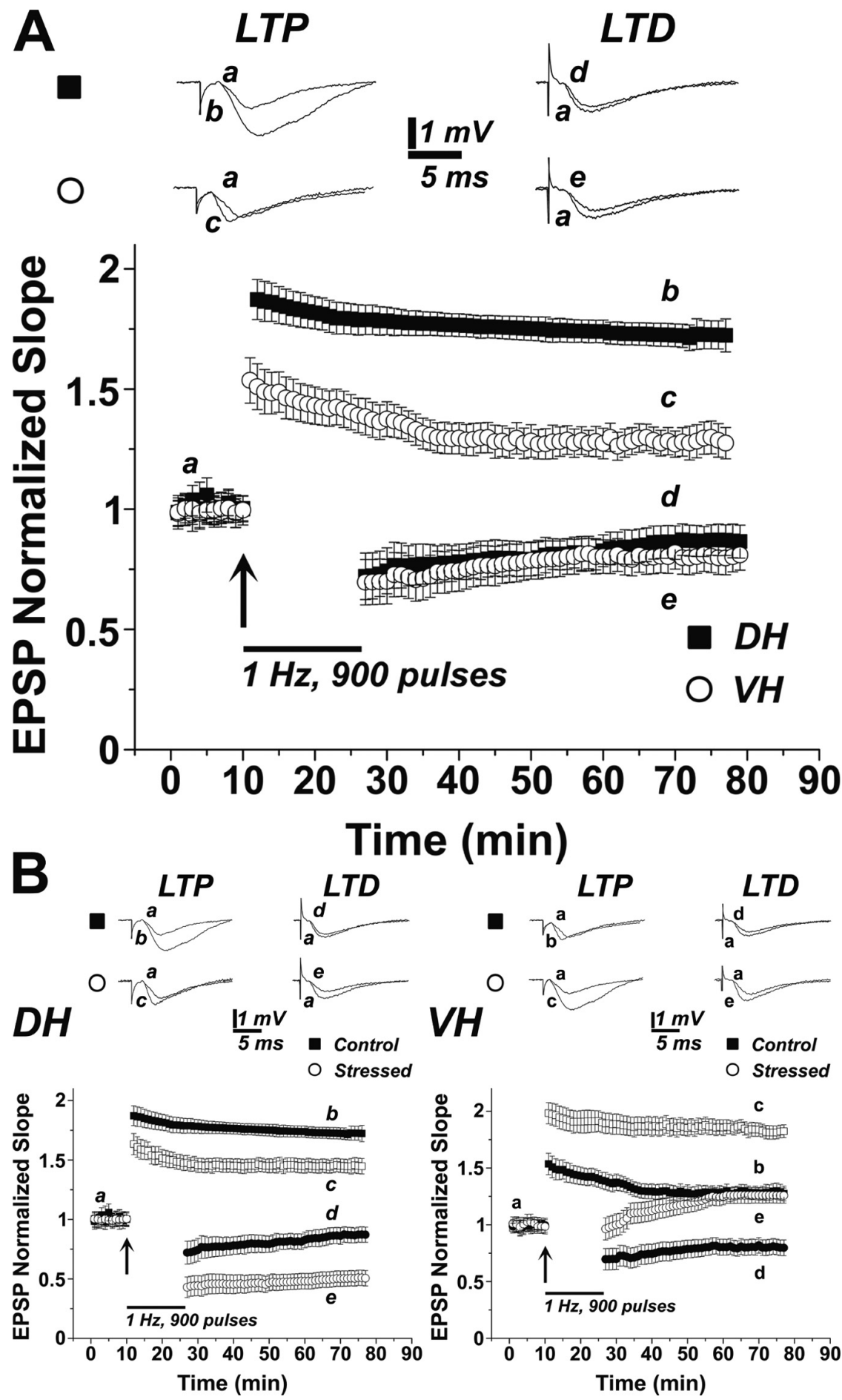

Figure 1. A similar LTD in the DH and VH is affected in an opposite manner by stress. $A$, LTP is larger in the DH than in the VH, but LTD is the same in both regions. In separate groups of slices, LTD and LTP were generated in both slice types. As seen previously (Maggio and Segal, 2007), LTP was larger in DH than in VH, but the two regions produced similar LTD (7 slices in each condition, sample traces above the grouped data illustrate records taken at the indicated time points). $\boldsymbol{B}$, Stress affects differently LTP and LTD in the DH and VH; while LTP is reduced, and LTD is enhanced in the DH, LTP in the VH is enhanced, but LTD is converted to slow-onset LTP (bottom right). Please note that the control groups in both the $\mathrm{DH}$ and VH are the same as in $\mathrm{A}$. Sample illustrations are at the indicated time points marked on the grouped data, which are averages of seven slices each. 


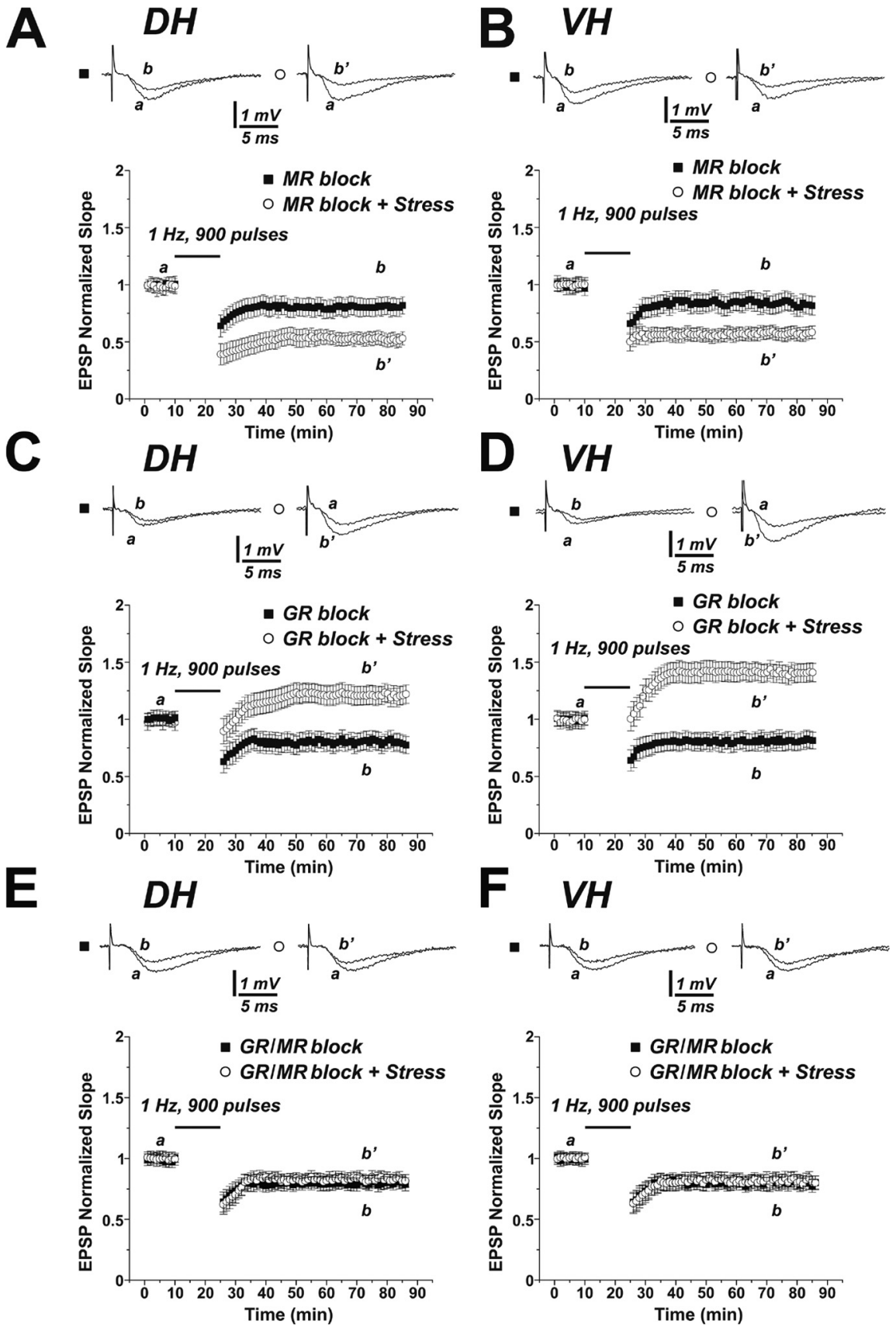

Figure 2. Blockade of MR, GR, or both eliminates stress-induced LTD differences between DH and VH. Comparisons were made between slices (7 each) taken from animals that were injected with the receptor antagonist before being stressed and controls that were injected with the antagonists but not stressed. Blockade of MRs (i.e., activation of only GRs) during stress produced enhanced LTD in both DH and VH $(\boldsymbol{A}, \boldsymbol{B})$, whereas blockade of GRs (i.e., activation of only MRs) converted LTD into LTP in both the DH and VH in the slices taken from the stressed rats $(\boldsymbol{C}, \boldsymbol{D})$. Blockade of both GRs and MRs blocked the effects of stress on LTD, which was now similar to drug-injected but unstressed rats $(\boldsymbol{E}, \boldsymbol{F})$.

examined the changes in reactivity to LTD-generating stimulation paradigm. Grouped data of the time course of the experiments are presented in Figure 2, and the summary of the statistical analysis of the data taken at a standard 5 min interval starting 40 min after the LTD train, in Figure 3. Quite surprisingly, while MR and GR antagonism did not affect LTD on their own in either the $\mathrm{DH}(\mathrm{MR}, 81.5 \pm 8 \%$, and GR, $79.2 \pm 7 \%)$ or the VH (MR, $86 \pm 7$, and GR, $79 \pm 7 \%$ of baseline levels) (Fig. $2 A-D$ ), they did modify the ability to express LTD following stress quite substantially, and in opposite directions. Thus, following stress, LTD in the $\mathrm{DH}$ amounted to $52.3 \pm 3 \%$ of baseline level in the presence of the MR antagonist, which is not much different from the effect of stress alone (see above and Fig. 3). On the other hand, in VH, LTD was formed anew in presence of the MR antagonist, reaching the level of $57.2 \pm 4 \%$ of baseline (Figs. 2 B, 3). Similar results were obtained when the LTD-inducing protocol involved two trains of 600 stimuli at $1 \mathrm{~Hz}$ (supplemental Fig. 2C,D, available at www. jneurosci.org as supplemental material).

In contrast, GR antagonism in stressed rats converted LTD in the DH into a slowonset LTP, reaching $121 \pm 4 \%$ of baseline level. In VH, the GR blockade in stressed rats caused a change in response to the stimulation up to $140.9 \pm 3 \%$ of baseline, which was not much different from the effect of stress alone in the VH. Thus, MR antagonism in stressed rats converted $\mathrm{VH}$ into DH-like behavior (LTD), whereas GR antagonism converted $\mathrm{DH}$ into $\mathrm{VH}$-like behavior (slow-onset LTP) in response to the same stimulation protocol (Fig. 3).

When the two corticosterone antagonists were applied together, the effects of stress and that of one antagonist by itself were canceled, and both the $\mathrm{DH}$ and $\mathrm{VH}$ produced the same level of LTD as in the nonstressed condition $(\mathrm{DH}, 83.3 \pm 4.2 \%$, $\mathrm{VH}, 81.6 \pm 6 \%$ of baseline, not different from nonstressed condition) (Figs. 2E, F, 3).

Acute effects of corticosterone agonists on LTD in DH and VH slices

Slices of $\mathrm{DH}$ and $\mathrm{VH}$ were recorded with two stimulating electrodes placed on both sides of the recording electrode in stratum radiatum of CA1 region (as in Maggio and Segal, 2007b). Before induction of LTD using the 900 pulse, $1 \mathrm{~Hz}$ protocol, responses of the slices was approximately the same for the two stimulating electrodes in both the DH and VH. Furthermore, LTD induction in one pathway did not affect the response to the other pathway (Fig. 4). Following establishment of LTD in one pathway, the slices were perfused continuously for at least $1 \mathrm{~h}$ with either dexamethasone, to activate GRs, or aldosterone, to activate MRs. In both the DH and VH (Fig. 4 , top), perfusion of either agonist did not change the baseline response to the stimulation in the two pathways studied. However, the activation of GRs caused a significant enhancement of a newly formed LTD, measured $30 \mathrm{~min}$ after the induction interval. In the DH, LTD amounted to $53 \pm 4 \%$ of baseline in presence of dexamethasone, compared with $75 \pm 5 \%$ in the control pathway $(p<0.01)$. In the $\mathrm{VH}$, the presence of dexamethasone enhanced LTD from $76 \pm 5 \%$ to $53 \pm 5 \%$ of baseline $(n=7$ slices each, $p<0.01)$.

Conversely, the presence of the MR agonist aldosterone (Fig. 4 , bottom) in DH slices reversed LTD from $76 \pm 5 \%$ of baseline 


\section{DH}
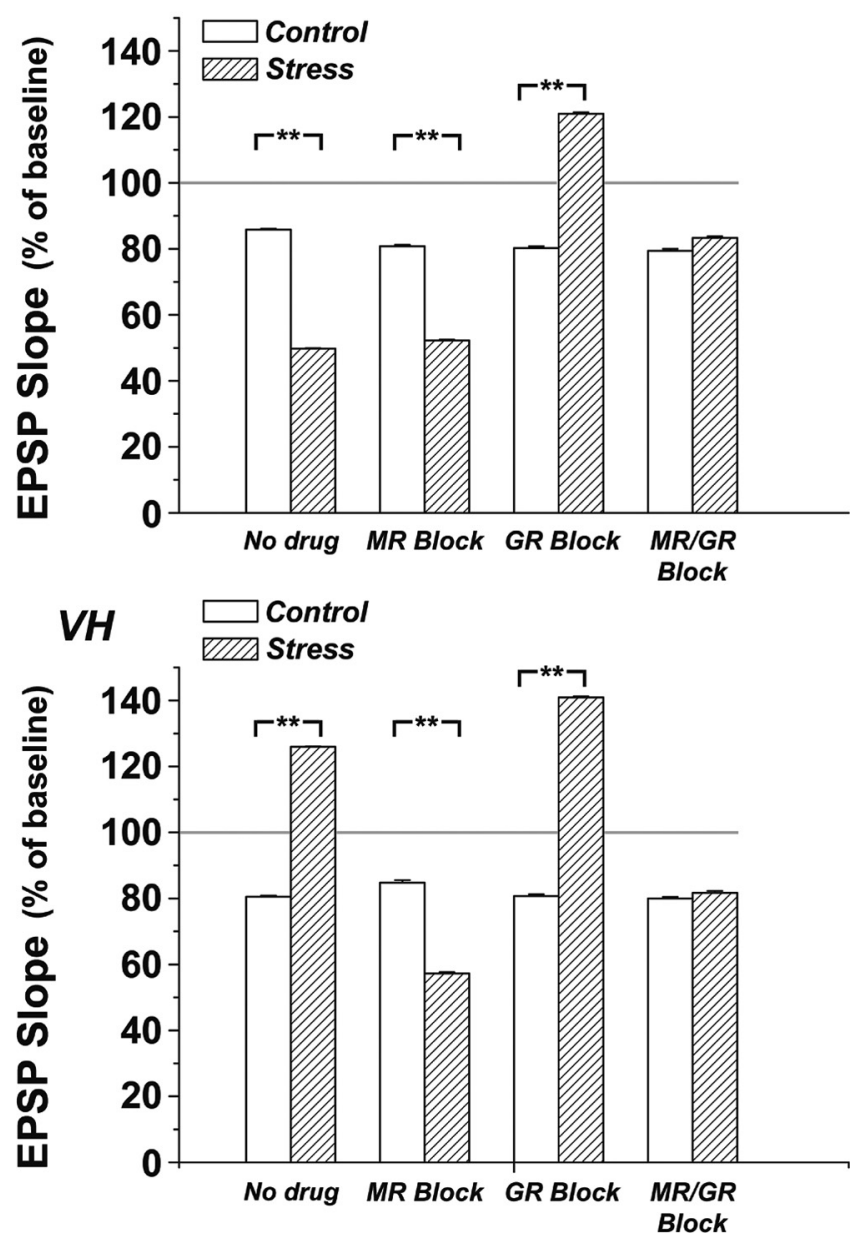

Figure 3. Summary of changes in EPSP slopes for all of the different groups. Control and swim stress conditions are compared with animals injected with antagonists for MR, GR, or both in the DH (top) and the VH (bottom). Results are based on a 5 min average taken at 40 min after a $1 \mathrm{~Hz}, 900$-pulse train of stimuli that produced LTD in the control condition, which was the same for both DH and VH. Two-way ANOVA on the averaged data in DH revealed a significant difference among the overall groups with respect to the pharmacological treatment $(F=11.67, p<$ 0.0001 ) and not with respect to the behavioral treatment (stress/control) the animals underwent $(F=1.17, p=0.28)$. However, post hoc $t$ test analysis run between control and stressed animals of the different pharmacological treatments group revealed a significant difference among stress/control, MR block/MR block plus stress, and GR block/GR block plus stress. In VH, two-way ANOVA analysis revealed a significant difference among the overall groups with respect to the pharmacological treatment $(F=12.28, p<0.0001)$ and the behavioral treatment (stress/control) $(F=14.02, p<0.001)$. Post hoc $t$ test analysis revealed a significant difference among stress/control, MR block/MR block plus stress, and GR block/GR block plus stress. Asterisks denote $p<0.001$.

to $125 \pm 4 \%$, resulting in a genuine LTP of reactivity to the stimulation in the particular pathway studied $(p<0.001)$. Likewise, in VH slices, LTD in the control pathway, amounting to $73 \pm 5 \%$ of baseline, was converted in the presence of aldosterone to a response that was $153 \pm 6 \%$ of baseline values $(p<0.0001)$. Interestingly, the potentiation of the response to afferent stimulation by the presence of aldosterone was larger in the $\mathrm{VH}$ than in the $\mathrm{DH}$, indicating that other factors may be involved in the plasticity of responses to the altered pattern of stimulation in the VH (see also Maggio and Segal, 2009).

\section{Discussion}

In the present study, we were able to replicate our earlier results (Maggio and Segal 2007a,b) showing that the VH CA1 expresses a smaller LTP than that produced in the DH. This deficit is not likely to be caused by specific synaptic properties in this region, since the basal synaptic transmission, expressed in the inputoutput relations, was similar in the different sectors (Maggio and Segal, 2007b). Surprisingly, both sectors of the hippocampus expressed similar magnitudes of LTD, indicating that the difference in LTP in control conditions is not likely to reflect a difference in modification threshold (Bear et al., 1987), but a genuine selective difference in LTP-generating mechanism.

The differential effect of stress on LTD in the $\mathrm{DH}$ and $\mathrm{VH}$ is striking indeed; starting from the same level of LTD in control conditions, stress enhanced LTD in the DH, while it converted LTD into slow-onset LTP in the VH. The effects of stress in the $\mathrm{DH}$ are blocked by GR antagonists, and in the VH by MR antagonists. These results have interesting mechanistic and functional implications in that they indicate that stress enhances LTP and suppresses LTD in VH, while it suppresses LTP and enhances LTD in DH. Together with our recent observations on the effects of stress and corticosterone on intrinsic properties of CA1 pyramidal neurons (Maggio and Segal, 2009), these steroid effects on LTP/LTD can be taken to indicate that stress affects neuronal excitability in the two regions of the hippocampus in an opposite manner, i.e., to increase excitability in the $\mathrm{VH}$ and reduce it in the DH. Regulation of excitability can be achieved by controlling intrinsic ionic conductances in the affected neurons or by controlling excitatory or inhibitory synaptic drive to these neurons. Earlier work has demonstrated that activation of steroid receptors can affect calcium conductances in hippocampal neurons, which will enhance the efficacy of synaptic input and may allow a larger LTP (Chameau et al., 2007; Maggio and Segal, 2007b; Joëls, 2008). In addition, steroid hormones enhance miniature excitatory synaptic currents (Karst and Joëls, 2005), which may also contribute to the enhanced synaptic excitability. Finally, our recent observations illustrate that corticosterone acting at an MR suppresses inhibitory synaptic currents, whereas at a GR it enhances inhibitory synaptic currents. In line with these observations, we now found that stress, in presence of blocked GRs causes enhancement of LTP and suppression of LTD in both the $\mathrm{DH}$ and $\mathrm{VH}$, and conversely, in presence of blocked MRs, stress causes enhancement of LTD in VH. These data are taken to indicate that the difference between $\mathrm{DH}$ and $\mathrm{VH}$ is in the relative contribution of GRs and MRs to the excitability of hippocampal neurons, as expressed in their reactivity to patterned stimulation. Indeed, recent observations indicate that there is a marked difference between $\mathrm{DH}$ and $\mathrm{VH}$ in the distribution of GRs and MRs (Robertson et al., 2005). Consequently, activation of GRs is more effective in the $\mathrm{DH}$ than in the $\mathrm{VH}$, and blockade of these receptors in the $\mathrm{DH}$ produces a response to the LTD-inducing $1 \mathrm{~Hz}$ stimulation akin to that produced in $\mathrm{VH}$ slices.

Our data also indicate that contrary to a previously proposed role of a sustained activation of the high-affinity MRs by ambient corticosterone (Joëls, 2006), ambient corticosterone did not have an effect on reactivity of the hippocampus to LTD-generating stimulation in our testing conditions, as the injection of either MR or GR antagonists had no effect on basal reactivity to the afferent stimulation, or on LTD in either DH and VH.

Corticosterone has two types of responses: a genomic and a nongenomic (de Kloet et al., 1996; Smythe et al., 1997; Yamada et al., 2003; Karst et al., 2005; Karst and Joëls, 2005). The first one requires protein synthesis and is activated by a low-affinity GR, hence is thought to be a slow response. The second one is mediated by a membrane-bound, high-affinity MR, and is considered to be a faster response. In our experiments, both antagonists were 

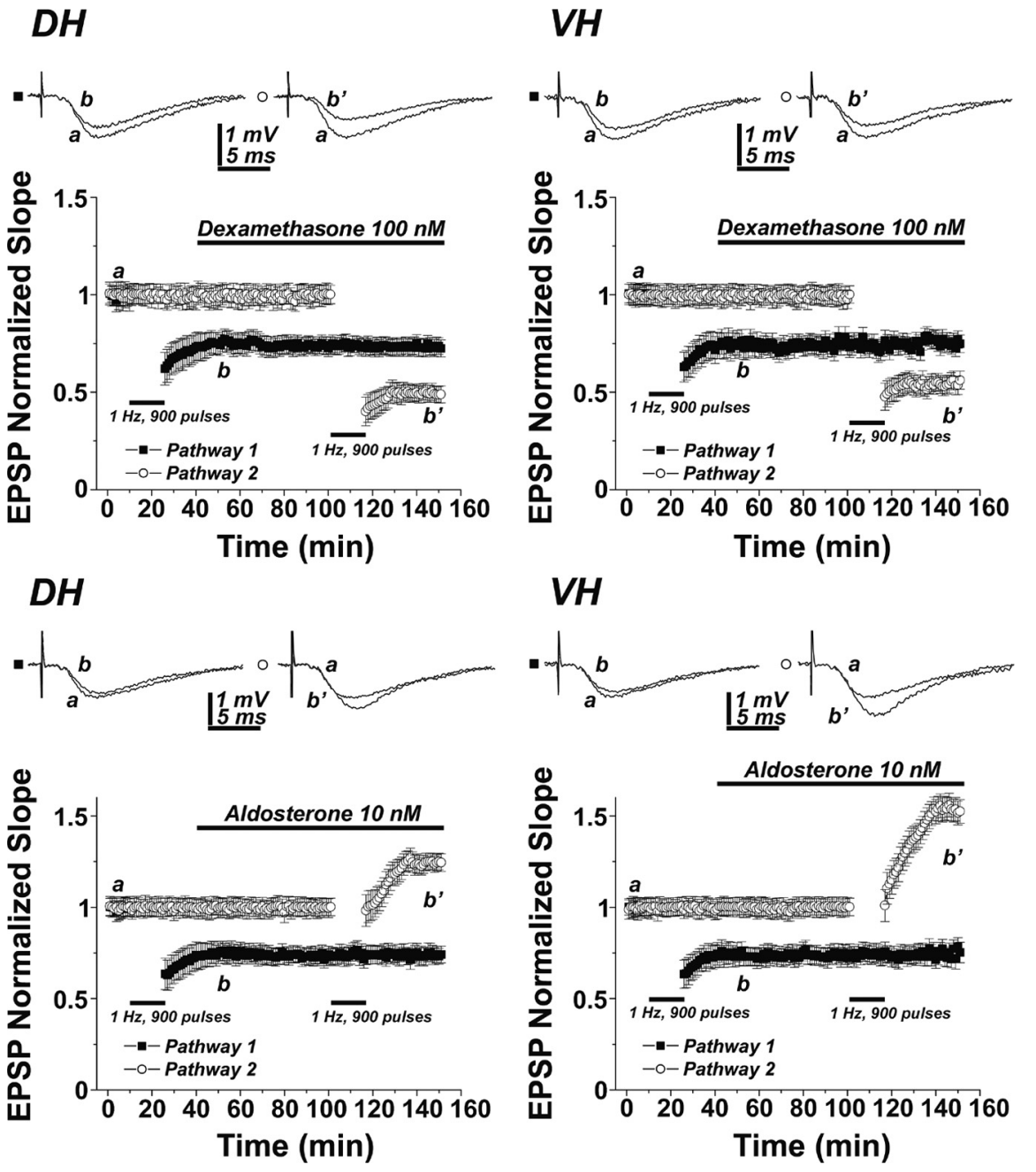

Figure 4. Acute exposure to corticosterone agonists mimic the effects of stress on LTD. Slices of DH (left column) and VH (right column) were recorded for $150 \mathrm{~min}$ each. The responses to two stimulating electrodes equidistant from the recording electrodes were recorded. Initially, one pathway was subjected to LTD-generating protocol, and after the response to this pathway was stabilized, the slices were exposed to either dexamethasone (top) or aldosterone (bottom) for $110 \mathrm{~min}$, during which time the other pathway was exposed to LTD induction protocol (bars). Sample illustrations of responses to stimulation of either pathway are presented above the averaged records, taken from seven slices each. In both DH and VH, dexamethasone enhanced LTD, while aldosterone converted LTD into a slow-onset LTP.

applied before the stressful experience, and hours before the recording session to produce opposite effects; hence it is likely that stress activates these two receptor species at the same time and that its action is long lasting.

The functional implications of our results can be related to previous studies on functional differences between the $\mathrm{DH}$, being associated with cognitive functions, including spatial maps and working memories, and the $\mathrm{VH}$, associated with emotional memories (Bannerman et al., 2003). Thus, under normal conditions, the $\mathrm{DH}$ connections with the neocortex are more amenable to modification and plasticity than the $\mathrm{VH}$ connections with the hypothalamus and the amygdala. This situation is reversed under stress, when the connections of the $\mathrm{VH}$ become more modifiable. One of the possible implications of this hypothesis is indicated in the behavioral analysis of the rats in the unavoidable water bath; blockade of MRs (activation of which enables the VH connections with the amygdala) causes rats to spend more time being immobile, hence helpless. The fact that stress modulates LTD differently in the $\mathrm{DH}$ and $\mathrm{VH}$ contributes to the functional distinction between these two regions of the hippocampus; by acti- vating selective corticosterone receptors, stress regulates the connectivity of the two hippocampal sectors with the rest of the brain. The functional implications of these suggestions need further exploration.

\section{References}

Bannerman DM, Grubb M, Deacon RM, Yee BK, Feldon J, Rawlins JN (2003) Ventral hippocampal lesions affect anxiety but not spatial learning. Behav Brain Res 139:197-213.

Bannerman DM, Rawlins JN, McHugh SB, Deacon RM, Yee BK, Bast T, Zhang WN, Pothuizen HH, Feldon J (2004) Regional dissociations within the hippocampus-memory and anxiety. Neurosci Biobehav Rev 28:273-283.

Bear MF, Cooper LN, Ebner FF (1987) A physiological basis for a theory of synapse modification. Science 237:42-48.

Chameau P, Qin Y, Spijker S, Smit G, Joëls M (2007) Glucocorticoids specifically enhance L-type calcium current amplitude and affect calcium channel subunit expression in the mouse hippocampus. J Neurophysiol 97:5-14.

Colgin LL, Kubota D, Jia Y, Rex CS, Lynch G (2004) Long-term potentiation is impaired in rat hippocampal slices that produce spontaneous sharp waves. J Physiol 558:953-961.

Colombo M, Fernandez T, Nakamura K, Gross CG (1998) Functional differentiation along the anterior-posterior axis of the hippocampus in monkeys. J Neurophysiol 80:1002-1005.

Degroot A, Treit D (2004) Anxiety is functionally segregated within the septo-hippocampal system. Brain Res 1001:60-71.

de Kloet ER, Rots NY, Cools AR (1996) Braincorticosteroid hormone dialogue: slow and persistent. Cell Mol Neurobiol 16:345-356.

Dudek SM, Bear MF (1992) Homosynaptic longterm depression in area CAl of hippocampus and effects of $\mathrm{N}$-methyl-D-aspartate receptor blockade. Proc Natl Acad Sci U S A 89:4363-4367.

Finn DP, Martí O, Harbuz MS, Vallès A, Belda X Márquez C, Jessop DS, Lalies MD, Armario A, Nutt DJ, Hudson AL (2003) Behavioral, neuroendocrine and neurochemical effects of the imidazoline I2 receptor selective ligand BU224 in naive rats and rats exposed to the stress of the forced swim test. Psychopharmacology 167:195-202.

Joëls M (2006) Corticosteroid effects in the brain: U-shape it. Trends Pharmacol Sci 27:244-250.

Joëls M (2008) Functional actions of corticosteroids in the hippocampus. Eur J Pharmacol 583:312-321.

Karst H, Joëls M (2005) Corticosterone slowly enhances miniature excitatory postsynaptic current amplitude in mice CA1 hippocampal cells. J Neurophysiol 94:3479-3486.

Karst H, Berger S, Turiault M, Tronche F, Schütz G, Joëls M (2005) Mineralocorticoid receptors are indispensable for nongenomic modulation of hippocampal glutamate transmission by corticosterone. Proc Natl Acad Sci U S A 102:19204-19207.

Kjelstrup KG, Tuvnes FA, Steffenach HA, Murison R, Moser EI, Moser MB (2002) Reduced fear expression after lesions of the ventral hippocampus. Proc Natl Acad Sci U S A 99:10825-10830.

Krugers HJ, Alfarez DN, Karst H, Parashkouhi K, van Gemert N, Joëls M (2005) Corticosterone shifts different forms of synaptic potentiation in opposite directions. Hippocampus 15:697-703.

Maggio N, Segal M (2007a) Unique regulation of long term potentiation in the rat ventral hippocampus. Hippocampus 17:10-25.

Maggio N, Segal M (2007b) Striking variations in corticosteroid modulation of long-term potentiation along the septotemporal axis of the hippocampus. J Neurosci 27:5757-5765. 
Maggio N, Segal M (2009) Differential corticosteroid modulation of inhibitory synaptic currents in the dorsal and ventral hippocampus. J Neurosci 29:2857-2866.

Maruki K, Izaki Y, Nomura M, Yamauchi T (2001) Differences in pairedpulse facilitation and long-term potentiation between dorsal and ventral CA1 regions in anesthetized rats. Hippocampus 11:655-661.

Moser MB, Moser EI (1998) Functional differentiation in the hippocampus. Hippocampus 8:608-619.

Papatheodoropoulos C, Kostopoulos G (2000) Decreased ability of rat temporal hippocampal CA1 region to produce long-term potentiation. Neurosci Lett 279:177-180.

Pothuizen HH, Zhang WN, Jongen-Rêlo AL, Feldon J, Yee BK (2004) Dissociation of function between the dorsal and the ventral hippocampus in spatial learning abilities of the rat: a within-subject, within-task comparison of reference and working spatial memory. Eur J Neurosci 19:705-712.

Robertson DA, Beattie JE, Reid IC, Balfour DJ (2005) Regulation of corticosteroid receptors in the rat brain: the role of serotonin and stress. Eur J Neurosci 21:1511-1520.
Smriga M, Nishiyama N, Saito H (1998) Mineralocorticoid receptormediated enhancement of neuronal excitability and synaptic plasticity in the dentate gyrus in vivo is dependent on the beta-adrenergic activity. J Neurosci Res 51:593-601.

Smythe JW, Murphy D, Timothy C, Costall B (1997) Hippocampal mineralocorticoid, but not glucocorticoid, receptors modulate anxiety-like behavior in rats. Pharmacol Biochem Behav 56:507-513.

Strange BA, Fletcher PC, Henson RN, Friston KJ, Dolan RJ (1999) Segregating the functions of human hippocampus. Proc Natl Acad Sci U S A 96:4034-4039.

Trivedi MA, Coover GD (2004) Lesions of the ventral hippocampus, but not the dorsal hippocampus, impair conditioned fear expression and inhibitory avoidance on the elevated T-maze. Neurobiol Learn Mem 81:172-184.

Yamada K, McEwen BS, Pavlides C (2003) Site and time dependent effects of acute stress on hippocampal long-term potentiation in freely behaving rats. Exp Brain Res 152:52-59. 